Georgian Mathematical Journal

Volume 12 (2005), Number 1, 189-200

\title{
WINTNER-TYPE OSCILLATION CRITERIA OF SEMILINEAR ELLIPTIC INEQUALITIES
}

\author{
ZHITING XU
}

\begin{abstract}
Wintner-type oscillation criteria of semilinear elliptic inequalities are obtained by using partial Riccati technique. The results presented improve the oscillation criteria due to E. S. Noussair and C.A. Swanson [3].
\end{abstract}

2000 Mathematics Subject Classification: 35B06, 35J60, 34C10.

Key words and phrases: Oscillation, semilinear elliptic inequalities, second order, partial Riccati technique.

\section{INTRODUCTION}

In this paper, we are concerned with obtaining Wintner-type oscillation criteria for semilinear elliptic inequalities

$$
(L y)(x)+q(x) f(y(x)) \leq 0, \quad x \in \Omega\left(r_{0}\right),
$$

where $\Omega\left(r_{0}\right)=\left\{x \in \mathbb{R}^{N}:|x| \geq r_{0}\right\}$ for $r_{0} \geq 0,|\cdot|$ is the usual Euclidean norm in $\mathbb{R}^{N}$, and $N \geq 2$.

Throughout this paper it is always assumed that the following hypotheses are valid without further mention.

$\left(H_{1}\right) L$ is an elliptic operator of the form

$$
L=\sum_{i, j=1}^{N} D_{i}\left[a_{i j}(x) D_{j}\right]+\sum_{i=1}^{N} b_{i}(x) D_{i}, \quad x \in \Omega\left(r_{0}\right),
$$

where $x=\left(x_{i}\right), D_{i}=\partial / \partial x_{i}, a_{i j} \in C_{l o c}^{1+\nu}\left(\Omega\left(r_{0}\right), \mathbb{R}\right), b_{i} \in C_{l o c}^{\nu}\left(\Omega\left(r_{0}\right), \mathbb{R}\right)$ for all $i, j$, $(\nu \in(0,1))$, and the symmetric matrix $A(x)=\left(a_{i j}\right)$ is positive definite at each $x \in \overline{\Omega\left(r_{0}\right)}$. Let $\lambda_{\max }(x) \in C\left(\Omega\left(r_{0}\right), \mathbb{R}\right)$ be the largest eigenvalue of the matrix $A(x)$. We suppose that there exists a function $\lambda \in C\left(\left[r_{0}, \infty\right), \mathbb{R}^{+}\right)$such that

$$
\lambda(r) \geq \max _{|x|=r} \lambda_{\max }(x), \quad \text { for } r \geq r_{0} ;
$$

$\left(H_{2}\right) \quad f \in C(\mathbb{R}, \mathbb{R}) \cup C^{1}(\mathbb{R}-\{0\}, \mathbb{R}), y f(y)>0$ and $f^{\prime}(y) \geq k>0$ for $y \neq 0 ;$ $\left(H_{3}\right) \quad q \in C_{l o c}^{\nu}\left(\Omega\left(r_{0}\right), \mathbb{R}\right),(\nu \in(0,1))$.

By a solution of $(1.1)$, we mean a function $y \in C_{l o c}^{2+\nu}\left(\Omega\left(r_{0}\right), \mathbb{R}\right)(\nu \in(0,1))$ satisfying (1.1) almost everywhere on $\Omega\left(r_{0}\right)$. For the question of the existence of a solution of (1.1) we refer the reader to the monograph [1]. Our attention is restricted to those solutions which do not vanish identically in any neighborhood of $|x|$. A nontrivial solution $y(x)$ of (1.1) is said to be oscillatory if, for any $R>0, y(x)$ has zero on $\Omega\left(r_{0}\right) \cap\{x:|x|>R\}$, otherwise it is said to be nonoscillatory. (1.1) is called oscillatory if all its solutions are oscillatory. 
In the qualitative theory of nonlinear partial differential equations, one of the important problems is to determine whether solutions of the equation under consideration are or are not oscillatory. A number of results on the oscillation of (1.1) are obtained by imposing restrictions on the elliptic operator $L$. An important special case of $(1.1)$ is the case $b_{i}(x) \equiv 0$ (for all $i$ ), for which (1.1) becomes

$$
\sum_{i, j=1}^{N} D_{i}\left[a_{i j}(x) D_{j} y\right]+q(x) f(y) \leq 0 .
$$

Concerning (1.2) there exists a well-elaborated oscillation theory. In 1980, Noussair and Swanson [3] first extended the well-known Wintner theorem [5] to (1.2) based on the partial Riccati transformation. The survey paper by Swanson [4] contains a complete bibliography till 1979. Recently, Xu [6] and Zhang et. al. [8] obtained Kamenev-type oscillation criteria [2]. On the other hand, using the averaging functions from a general class of parameter functions, $\mathrm{Xu}[7]$ gave new oscillation criteria for (1.2). It seems, however, that a very few results are established for (1.1) in general form. Motivated by this fact, in this paper we develop the technique exploited by Noussair and Swanson [3] to establish Wintner-type oscillation criteria for (1.1), which extend and improve the results in [3]. Finally, some examples are given to illustrate the advantages of our results. It is to be emphasized that we do not assume any condition on the functions $b_{i}$ and $q$ except the conditions $b_{i}, q \in C_{l o c}^{\nu}(\Omega)$ (for all $i$ ). The results obtained here are new even for (1.2).

\section{MAin Results}

Lemma 2.1. Let $\alpha, \beta \in \mathbb{R}^{N}, C>0$, then

$$
C \alpha \alpha^{T}+\alpha \beta^{T} \geq \frac{C}{2} \alpha \alpha^{T}-\frac{1}{2 C} \beta \beta^{T} .
$$

Lemma 2.1 is easy to verify and the proof is omitted.

We now introduce our principal notations. For any given functions $\rho \in$ $C^{1}\left(\left[r_{0}, \infty\right), \mathbb{R}^{+}\right)$and $(\lambda \eta) \in C^{1}\left(\left[r_{0}, \infty\right), \mathbb{R}\right)$, we define

$$
g(r)=\frac{k}{2 \omega} \frac{r^{1-N}}{\lambda(r) \rho(r)}, \quad p(r)=\frac{\rho^{\prime}(r)}{\rho(r)}+\frac{k}{\omega} \eta(r) r^{1-N},
$$

and

$$
\begin{aligned}
\theta(r)= & \rho(r)\left\{\int_{S_{r}}\left[q(x)-\frac{1}{2 k} \lambda(x)\left|B^{T} A^{-1}\right|^{2}\right] d \sigma+\frac{k}{2 \omega} r^{1-N} \lambda(r) \eta^{2}(r)\right. \\
& \left.-[\lambda(r) \eta(r)]^{\prime}\right\}+\frac{1}{2}\left(\frac{p(r)}{g(r)}\right)^{\prime}-\frac{p^{2}(r)}{4 g(r)},
\end{aligned}
$$

where $S_{r}=\left\{x \in \mathbb{R}^{N}:|x|=r\right\}, \omega$ and $d \sigma$ denote the surface measure of unit sphere and the spherical integral element in $\mathbb{R}^{N}$, respectively, and $B^{T}=$ $\left(b_{1}(x), \ldots, b_{N}(x)\right)$. 
Theorem 2.1. Suppose that there exist functions $\rho \in C^{1}\left(\left[r_{0}, \infty\right), \mathbb{R}^{+}\right)$, $(\lambda \eta) \in C^{1}\left(\left[r_{0}, \infty\right), \mathbb{R}\right)$, satisfying

$$
\int_{r_{0}}^{\infty} g(r) d r=\infty
$$

and

$$
\int_{r_{0}}^{\infty} \theta(r) d r=\infty
$$

then (1.1) is oscillatory.

Proof. Let $y=y(x)$ be a nonoscillatory solution of (1.1). Without loss of generality we assume that $y(x)>0$ for $|x|>r_{0}$. Put

$$
W(x)=\frac{1}{f(y(x))}\left(A^{\nabla} y\right)(x)
$$

where ${ }^{\nabla} y$ denotes the gradient of $y$. Differentiation of the $i$-th component of $W(x)$ with respect to $x_{i}$ gives

$$
D_{i} W(x)_{i}=-\frac{f^{\prime}(y)}{f^{2}(y)} D_{i} y\left[\sum_{j=1}^{N} a_{i j}(x) D_{j} y\right]+\frac{1}{f(y)} D_{i}\left[\sum_{j=1}^{N} a_{i j}(x) D_{j} y\right]
$$

for all $i$. Summation over $i$ and the use of (1.1) lead to

$$
\begin{aligned}
\operatorname{div} W(x) & \leq-f^{\prime}(y)\left(W^{T} A^{-1} W\right)(x)-\left(B^{T} A^{-1} W\right)(x)-q(x) \\
& \leq-k\left(W^{T} A^{-1} W\right)(x)-\left(B^{T} A^{-1} W\right)(x)-q(x) \quad\left(b y\left(H_{2}\right)\right) \\
& \leq-\frac{k}{\lambda(x)}\left(W^{T} W\right)(x)-\left(B^{T} A^{-1} W\right)(x)-q(x) \quad\left(\text { by }\left(H_{1}\right)\right) \\
& \leq-\frac{k}{2 \lambda(x)}\left(W^{T} W\right)(x)+\frac{1}{2 k} \lambda(x)\left|B^{T} A^{-1}\right|^{2}-q(x)
\end{aligned}
$$

(by Lemma 2.1).

Let

$$
w(r)=\rho(r)\left[\int_{S_{r}} W(x) \cdot \nu(x) d \sigma+\lambda(r) \eta(r)\right] \quad \text { for } \quad r \geq r_{0},
$$

where $\nu(x)=x /|x|,(|x| \neq 0)$, denotes the outward unit normal. Using the divergence theorem in (2.5), we obtain by (2.4)

$$
\begin{aligned}
w^{\prime}(r) & =\frac{\rho^{\prime}(r)}{\rho(r)} w(r)+\rho(r)\left\{\int_{S_{r}} \operatorname{div} W(x) d \sigma+[\lambda(r) \eta(r)]^{\prime}\right\} \\
& \leq \frac{\rho^{\prime}(r)}{\rho(r)} w(r)-\rho(r)\left\{\frac{k}{2 \lambda(r)} \int_{S_{r}}\left(W^{T} W\right)(x) d \sigma\right.
\end{aligned}
$$




$$
\left.+\int_{S_{r}}\left[q(x)-\frac{1}{2 k} \lambda(x)\left|B^{T} A^{-1}\right|^{2}\right] d \sigma-[\lambda(r) \eta(r)]^{\prime}\right\} .
$$

By the Schwartz inequality

$$
\int_{S_{r}}|W(x)|^{2} d \sigma \geq \frac{r^{1-N}}{\omega}\left[\int_{S_{r}} W(x) \cdot \nu(x) d \sigma\right]^{2} .
$$

Thus for $r \geq r_{0}$

$$
\begin{aligned}
w^{\prime}(r) \leq & \frac{\rho^{\prime}(r)}{\rho(r)} w(r)-\rho(r)\left\{\frac{k}{2 \omega} \frac{r^{1-N}}{\lambda(r)}\left[\int_{S_{r}} W(x) \cdot \nu(x) d \sigma\right]^{2}\right. \\
& \left.+\int_{S_{r}}\left[q(x)-\frac{1}{2 k} \lambda(x)\left|B^{T} A^{-1}\right|^{2}\right] d \sigma-[\lambda(r) \eta(r)]^{\prime}\right\} \\
= & \frac{\rho^{\prime}(r)}{\rho(r)} w(r)-\rho(r)\left\{\frac{k}{2 \omega} \frac{r^{1-N}}{\lambda(r)}\left[\frac{w(r)}{\rho(r)}-\lambda(r) \eta(r)\right]^{2}\right. \\
& \left.+\int_{S_{r}}\left[q(x)-\frac{1}{2 k} \lambda(x)\left|B^{T} A^{-1}\right|^{2}\right] d \sigma-[\lambda(r) \eta(r)]^{\prime}\right\} \\
= & -g(r) w^{2}(r)+p(r) w(r)-\rho(r)\left\{\int_{S_{r}}\left[q(x)-\frac{1}{2 k} \lambda(x)\left|B^{T} A^{-1}\right|^{2}\right] d \sigma\right. \\
& \left.+\frac{k}{2 \omega} r^{1-N} \lambda(r) \eta^{2}(r)-[\lambda(r) \eta(r)]^{\prime}\right\},
\end{aligned}
$$

that is

$$
Z^{\prime}(r) \leq-g(r) Z^{2}(r)-\theta(r)
$$

where

$$
Z(r)=w(r)-\frac{1}{2} \frac{p(r)}{g(r)}
$$

Hence, for all $r \geq r_{0}$, we have

$$
Z(r) \leq Z\left(r_{0}\right)-\int_{r_{0}}^{r} g(s) Z^{2}(s) d s-\int_{r_{0}}^{r} \theta(s) d s .
$$

By (2.3) and (2.7) we can find a number $a \geq r_{0}$ such that for all $r \geq a$

$$
Z(r) \leq-\int_{r_{0}}^{r} g(s) Z^{2}(s) d s=:-H(r)
$$

Thus

$$
H^{\prime}(r)=g(r) Z^{2}(r) \geq g(r) H^{2}(r)
$$


This yields

$$
\int_{r_{0}}^{r} g(s) d s \leq \int_{r_{0}}^{r} \frac{d H(r)}{H^{2}(r)} \leq \frac{1}{H\left(r_{0}\right)} \quad \text { for } \quad r \geq r_{0},
$$

which contradicts $(2.2)$, and this completes the proof.

Remark 2.1. For (1.2), let $\eta(r) \equiv 0$, then Theorem 2.1 improves Theorem 4 in $[3]$.

The following oscillation criteria (Theorems 2.2-2.4) treat the cases when it is not possible to verify easily conditions (2.2) or (2.3).

Theorem 2.2. Suppose that there exist functions $\rho \in C^{1}\left(\left[r_{0}, \infty\right), \mathbb{R}^{+}\right),(\lambda \eta) \in$ $C^{1}\left(\left[r_{0}, \infty\right), \mathbb{R}\right), \varphi \in C(\mathbb{R},[0, \infty))$ with $\varphi$ nondecreasing on $[0, \infty)$, and $\phi \in$ $C\left(\left[r_{0}, \infty\right), \mathbb{R}^{+}\right)$satisfying

$$
\begin{aligned}
\int_{r_{0}}^{\infty}\left[\int_{r_{0}}^{s} \frac{\phi^{2}(\tau)}{g(\tau)} d \tau\right]^{-1} \phi(s) \varphi\left(\int_{r_{0}}^{s} \phi(\tau) d \tau\right) d s=\infty \\
\int_{r_{0}}^{\infty} \frac{\varphi(s)}{s^{2}} d s<\infty
\end{aligned}
$$

If

$$
\lim _{r \rightarrow \infty}\left[\int_{r_{0}}^{r} \phi(s) d s\right]^{-1} \int_{r_{0}}^{r} \phi(s) \int_{r_{0}}^{s} \theta(\tau) d \tau d s=\infty
$$

then (1.1) is oscillatory.

Proof. By using the same argument as in the proof of Theorem 2.1, we get (2.7) holds for $r \geq r_{0}$. Multiplying (2.7) by $\phi(s)$ and integrating from $r_{0}$ to $r$, we have

$$
\begin{aligned}
& \int_{r_{0}}^{r} \phi(s) Z(s) d s+G(r) \\
& \quad \leq\left[\int_{r_{0}}^{r} \phi(s) d s\right]\left[Z\left(r_{0}\right)-\left(\int_{r_{0}}^{r} \phi(s) d s\right)^{-1} \int_{r_{0}}^{r} \phi(s) \int_{r_{0}}^{s} \theta(\tau) d \tau d s\right],
\end{aligned}
$$

where $G(r)=\int_{r_{0}}^{r} \phi(s) \int_{r_{0}}^{s} g(\tau) Z^{2}(\tau) d \tau d s$. In view of condition (2.10), there exists a constant $a>r_{0}$ such that

$$
Z\left(r_{0}\right)-\left[\int_{r_{0}}^{r} \phi(s) d s\right]^{-1} \int_{r_{0}}^{r} \phi(s) \int_{r_{0}}^{s} \theta(\tau) d \tau d s<0, \quad \text { for all } \quad r \geq a
$$


So, for every $r \geq a$

$$
G(r) \leq-\int_{r_{0}}^{r} \phi(s) Z(s) d s .
$$

In virtue of the Schwartz inequality

$$
G^{2}(r) \leq\left[\int_{r_{0}}^{r} \phi(s) Z(s) d s\right]^{2} \leq\left[\int_{r_{0}}^{r} \frac{\phi^{2}(s)}{g(s)} d s\right]\left[\int_{r_{0}}^{r} g(s) Z^{2}(s) d s\right],
$$

that is

$$
\left[\int_{r_{0}}^{r} \frac{\phi^{2}(s)}{g(s)} d s\right]^{-1} \phi(r) \leq \frac{G^{\prime}(r)}{G^{2}(r)}
$$

But, for all $r \geq a$

$$
G(r) \geq\left[\int_{a}^{r} \phi(s) d s\right]\left[\int_{r_{0}}^{a} g(s) Z^{2}(s) d s\right]=C \int_{a}^{r} \phi(s) d s,
$$

where $C=\int_{r_{0}}^{a} g(s) Z^{2}(s) d s$. Since $g(s) Z^{2}(s)$ is continuous and not identically zero on $\left[r_{0}, a\right]$, we have $C>0$. Thus, for all $r \geq a$,

$$
\varphi\left(\int_{a}^{r} \phi(s) d s\right) \leq \varphi\left(C^{-1} G(r)\right) .
$$

From (2.11) and (2.12) it follows that

$$
\begin{aligned}
& \int_{a}^{r}\left[\int_{r_{0}}^{s} \frac{\phi^{2}(\tau)}{g(\tau)} d \tau\right]^{-1} \phi(s) \varphi\left(\int_{a}^{s} \phi(\tau) d \tau\right) d s \\
& \quad \leq \int_{a}^{r} \frac{\varphi\left(C^{-1} G(s)\right)}{G^{2}(s)} d G(s)=\int_{C^{-1} G(a)}^{C^{-1} G(r)} \frac{\varphi(s)}{s^{2}} d s<\infty,
\end{aligned}
$$

which contradicts condition (2.8).

Lemma 2.2. Suppose that there exist functions $\rho \in C^{1}\left(\left[r_{0}, \infty\right), \mathbb{R}\right)$ and $(\lambda \eta) \in C^{1}\left(\left[r_{0}, \infty\right), \mathbb{R}\right)$ such that $(2.2)$ and

$$
\Theta(r):=\int_{r}^{\infty} \theta(r) d r<\infty \text { for } r \geq r_{0},
$$

hold. If (1.1) is nonoscillatory, then there exist a constant $a>r_{0}$ and a function $Z \in C^{1}([a, \infty), \mathbb{R})$ satisfying

$$
Z(r) \geq \int_{r}^{\infty} \theta(s) d s+\int_{r}^{\infty} g(s) Z^{2}(s) d s \quad \text { for } \quad r \geq a .
$$


Proof. Proceeding as in the proof of Theorem 2.1, there exist a number $a>r_{0}$ and a function $Z \in C^{1}([a, \infty), \mathbb{R})$ satisfying (2.6). Therefore for $b \geq r \geq a$

$$
Z(b)+\int_{r}^{b} \theta(\tau) d \tau+\int_{r}^{b} g(s) Z^{2}(s) d s \leq Z(r) .
$$

Now we claim that

$$
\int_{r}^{\infty} g(s) Z^{2}(s) d s<\infty
$$

Otherwise

$$
\int_{r}^{\infty} g(s) Z^{2}(s) d s=\infty
$$

then there is a number $a_{1} \geq a$ such that, taking into account (2.13) and (2.15),

$$
Z(b) \leq-\int_{a_{1}}^{b} g(s) Z^{2}(s) d s \quad \text { for } \quad b \geq a_{1}
$$

As in the proof of Theorem 2.1, it is easy to show that

$$
\int_{a_{1}}^{\infty} g(s) d s<\infty
$$

which contradicts (2.2). Thus (2.16) holds. Therefore, from (2.15), for $r \geq a$

$$
Z(r) \geq \limsup _{b \rightarrow \infty} Z(b)+\int_{r}^{\infty} \theta(s) d s+\int_{r}^{\infty} g(s) Z^{2}(s) d s .
$$

If $\limsup _{r \rightarrow \infty} Z(b)<0$, then there exist two numbers $\delta<0$ and $a_{2} \geq a_{1}$ such that $Z(b)<\delta$ for $b \geq a_{2}$. It follows from (2.2) that, for $r \geq b$,

$$
\int_{r}^{\infty} g(s) Z^{2}(s) d s \geq \delta^{2} \int_{r}^{\infty} g(s) d s=\infty
$$

which contradicts (2.16). Thus $\lim _{\sup _{b \rightarrow \infty}} Z(b) \geq 0$. It follows from (2.17) that (2.14) holds.

Theorem 2.3. Suppose that there exist functions $\rho \in C^{1}\left(\left[r_{0}, \infty\right), \mathbb{R}^{+}\right)$and $(\lambda \eta) \in C^{1}\left(\left[r_{0}, \infty\right), \mathbb{R}\right)$ such that $(2.2)$ and $(2.13)$ hold. If

$$
\int_{r_{0}}^{\infty} g(s) \Theta_{+}^{2}(s) \exp \left[2 \int_{r_{0}}^{s} g(\tau) \Theta(\tau) d \tau\right] d s=\infty,
$$

then equation (1.1) is oscillatory. 
Proof. Suppose that (1.1) is nonoscillatory. Then it follows from Lemma 2.2 that there exist a constant $a>r_{0}$ and a function $Z \in C^{1}([a, \infty), \mathbb{R})$ satisfying (2.14) for $r \geq a$. Define

$$
v(r)=\int_{r}^{\infty} g(s) Z^{2}(s) d s
$$

then

$$
v^{\prime}(r)=-g(r) Z^{2}(r) .
$$

Multiplying (2.19) by $\exp \left[2 \int_{r}^{s} g(\tau) \Theta(\tau) d \tau\right]$ and integrating from $r$ to $b$, we obtain

$$
\begin{aligned}
v(r)= & \exp \left[2 \int_{r}^{b} g(s) \Theta(s) d s\right] v(b) \\
& +\int_{r}^{b} g(s)\left[Z^{2}(s)-2 \Theta(s) v(s)\right] \exp \left[2 \int_{r}^{s} g(\tau) \Theta(\tau) d \tau\right] d s .
\end{aligned}
$$

It follows from (2.14) that $Z(r) \geq \Theta(r)+v(r)$ for $r \geq a$, which implies $Z^{2}(r)-$ $2 \Theta(r) v(r) \geq \Theta_{+}^{2}(r)+v^{2}(r)$ for $r \geq a$. This and (2.20) imply

$$
\begin{aligned}
v(r) \geq & \int_{r}^{\infty} g(s) \Theta_{+}^{2}(s) \exp \left[2 \int_{r}^{s} g(\tau) \Theta(\tau) d \tau\right] d s \\
& +\int_{r}^{\infty} g(s) v^{2}(s) \exp \left[2 \int_{r}^{s} g(\tau) \Theta(\tau) d \tau\right] d s .
\end{aligned}
$$

This contradicts (2.18). The proof is completed.

Theorem 2.4. Suppose that there exist functions $\rho \in C^{1}\left(\left[r_{0}, \infty\right), \mathbb{R}^{+}\right)$and $(\lambda \eta) \in C^{1}\left(\left[r_{0}, \infty\right), \mathbb{R}\right)$ such that $(2.2),(2.13)$ and

$$
\Theta_{1}(r):=\int_{r}^{\infty} g(s) \Theta_{+}^{2}(s) \exp \left[2 \int_{r}^{s} g(\tau) \Theta(\tau) d \tau\right] d s<\infty \quad \text { for } \quad r \geq r_{0}
$$

hold. If

$$
\int_{r_{0}}^{\infty} g(s) \Theta_{1}^{2}(s) \exp \left[2 \int_{r_{0}}^{s} g(\tau)\left(\Theta(\tau)+\Theta_{1}(\tau)\right) d \tau\right] d s=\infty
$$

then (1.1) is oscillatory. 
Proof. Proceeding as in the proof of Theorem 2.3, we can find a constant $a>r_{0}$ and a function $v \in C^{1}([a, \infty), \mathbb{R})$ satisfying $(2.21)$ for all $r \geq a$. Define

$$
u(r)=\int_{r}^{\infty} g(s) v^{2}(s) \exp \left[2 \int_{r}^{s} g(\tau) \Theta(\tau) d \tau\right] d s .
$$

Then

$$
\begin{aligned}
u^{\prime}(r) & =-g(r)\left[v^{2}(r)+2 \Theta(r) u(r)\right] \\
& \leq-g(r)\left\{\left[\Theta_{1}^{2}(r)+u^{2}(r)\right]+2\left[\Theta(r)+\Theta_{1}(r)\right] u(r)\right\} .
\end{aligned}
$$

Using $\exp \left[2 \int_{r}^{s} g(\tau)\left[\Theta(\tau)+\Theta_{1}(\tau)\right] d \tau\right.$ as an integrating factor, we integrate (2.23) from $r$ to $b$ and obtain

$$
\begin{aligned}
u(r) \geq & \exp \left[2 \int_{r}^{b} g(s)\left(\Theta(s)+\Theta_{1}(s)\right) d s\right] u(b) \\
& +\int_{r}^{b} g(s)\left[\Theta_{1}^{2}(s)+u^{2}(s)\right] \exp \left[2 \int_{r}^{s} g(\tau)\left(\Theta(\tau)+\Theta_{1}(\tau)\right) d \tau\right] d s \\
\geq & \int_{r}^{b} g(s) \Theta_{1}^{2}(s) \exp \left[2 \int_{r}^{s} g(\tau)\left(\Theta(\tau)+\Theta_{1}(\tau)\right) d \tau\right] d s \\
& +\int_{r}^{b} g(s) u^{2}(s) \exp \left[2 \int_{r}^{s} g(\tau)\left(\Theta(\tau)+\Theta_{1}(\tau)\right) d \tau\right] d s .
\end{aligned}
$$

Letting $b \rightarrow \infty$ in the above inequality, we get a contradiction to (2.22). This completes the proof.

Remark 2.2. If we assume further that $\Theta(r), \Theta_{1}(r), \ldots$ are integrable, similarly to Theorems 2.3 and 2.4, we can establish a number of oscillation criteria for equation(1.1).

\section{Corollaries AND Examples}

The results in Section 2 are of high degree of generality. With an appropriate choice of the functions $\rho, \eta$ and $\varphi$. Some interesting corollaries can be obtain from Theorems 2.1-2.4, for example.

The following is an oscillation result for the linear equations

$$
\Delta y+\sum_{i=1}^{N} b_{i}(x) \frac{\partial y}{\partial x_{i}}+q(x) y=0, \quad x \in \Omega\left(r_{0}\right),
$$

where $b_{i}, q \in C_{l o c}^{\nu}(\Omega), \nu \in C(0,1)$. 
Corollary 3.1. Suppose that the conditions

$$
\lim _{r \rightarrow \infty} \int_{r_{0}}^{r}\left[s \ln s q_{M}(s)-\frac{1}{2 s \ln s}\right] d s=\infty, \quad N=2,
$$

and

$$
\lim _{r \rightarrow \infty} \int_{r_{0}}^{r}\left[s q_{M}(s)-\frac{(2-N)^{2}}{2 s}\right] d s=\infty, \quad N \geq 3
$$

where

$$
q_{M}(r)=\frac{1}{\omega r^{N-1}} \int_{S_{r}}\left[q(x)-\frac{1}{2} \sum_{i=1}^{N} b_{i}^{2}(x)\right] d \sigma,
$$

hold. Then (3.1) is oscillatory.

Proof. The assertion of Corollary 3.1 follows from that of Theorem 2.1 if we choose $\eta(r) \equiv 0$ and

$$
\rho(r)=\left\{\begin{array}{cl}
\ln r, & \text { for } \quad N=2 \\
r^{2-N}, & \text { for } \quad N \geq 3
\end{array}\right.
$$

Remark 3.1. Corollary 3.1 improves some results in $[3,4]$.

Example 3.1. Consider the elliptic equation

$$
\Delta y+\frac{\sin |x|}{|x|} \frac{\partial y}{\partial x_{1}}+\frac{\cos |x|}{|x|} \frac{\partial y}{\partial x_{2}}+\frac{\mu}{|x|^{2}} y=0,
$$

for $|x| \geq e$, where $\mu$ is a constant with $2 \mu>1$, and $N=2$.

A direct calculation gives

$$
\begin{aligned}
& \int_{e}^{r}\left[s \ln s q_{M}(s)-\frac{1}{2 s \ln s}\right] d s \\
& \quad=\frac{2 \mu-1}{2} \ln ^{2} r-\frac{1}{2} \ln (\ln r)-\frac{2 \mu-1}{2} \rightarrow \infty \quad \text { as } \quad r \rightarrow \infty .
\end{aligned}
$$

Hence (3.4) is oscillatory by Corollary 3.1.

Example 3.2. Consider the elliptic equation

$$
\frac{\partial}{\partial x_{1}}\left(\frac{1}{|x|^{2}} \frac{\partial y}{\partial x_{1}}\right)+\frac{\partial}{\partial x_{2}}\left(\frac{1}{|x|^{2}} \frac{\partial y}{\partial x_{2}}\right)+\frac{2+\cos |x|-2|x| \sin |x|}{4|x|^{\frac{5}{2}}}(4 y+\sqrt[3]{y})=0
$$

for $|x| \geq e$, where $N=2$.

Here, we choose $\rho(r)=r, \eta(r)=-2 \pi, \phi(r)=1 / r$, and $\varphi(r)=\sqrt{r}$; then

$$
p(r)=0, \quad g(r)=\frac{1}{\pi}, \quad \theta(r)=\frac{\pi(2+\cos r-2 r \sin r)}{2 \sqrt{r}},
$$




$$
\begin{gathered}
\int_{e}^{r} \theta(s) d s=\pi[\sqrt{r}(2+\cos r)-\sqrt{e}(2+\cos e)] \geq \sqrt{r} \text { for } r \geq e \\
\lim _{r \rightarrow \infty}\left[\int_{e}^{r} \phi(s) d s\right]^{-1} \int_{e}^{r} \phi(s) \int_{e}^{s} \theta(\tau) d \tau d s \geq \lim _{r \rightarrow \infty} \frac{1}{\ln r} \int_{e}^{r} s^{-\frac{1}{2}} d s=\infty \\
\lim _{r \rightarrow \infty} \int_{e}^{r}\left[\int_{e}^{s} \frac{\phi^{2}(\tau)}{g(\tau)} d \tau\right]^{-1} \phi(s) \varphi\left(\int_{e}^{s} \phi(\tau) d \tau\right) d s \\
=\lim _{r \rightarrow \infty} \frac{e}{\pi} \int_{e}^{r} \frac{(\ln s-1)^{1 / 2}}{s-e} d s=\infty
\end{gathered}
$$

Thus, all conditions of Theorem 2.2 are satisfied and hence (3.5) is oscillatory.

Example 3.3. Consider the elliptic equation

$$
\frac{\partial}{\partial x_{1}}\left(\frac{1}{|x|} \frac{\partial y}{\partial x_{1}}\right)+\frac{\partial}{\partial x_{2}}\left(\frac{1}{|x|} \frac{\partial y}{\partial x_{2}}\right)+\frac{1}{|x|^{2}}\left(\frac{\partial y}{\partial x_{1}}+\frac{\partial y}{\partial x_{2}}\right)+\frac{v}{|x|^{3}}\left(y+y^{3}\right)=0
$$

for $|x| \geq 1$, where $v$ is a constant with $v \geq 7 / 2$ and $N=2$.

Taking $\rho(r)=1 / r, \eta(r)=2 \pi$, we have

$$
\begin{gathered}
p(r)=0, \quad g(r)=\frac{r}{4 \pi}, \quad \theta(r)=\frac{\pi(2 v+1)}{r^{3}}, \\
\Theta(r)=\int_{r}^{\infty} \theta(s) d s=\frac{\pi(2 v+1)}{2} \frac{1}{r^{2}}, \\
\lim _{r \rightarrow \infty} \int_{1}^{r} g(s) \Theta_{+}^{2}(s) \exp \left[2 \int_{1}^{s} g(\tau) \Theta(\tau) d \tau\right] d s \\
=\lim _{r \rightarrow \infty} \frac{(2 v+1)^{2} \pi}{16} \int_{1}^{r} s^{\frac{2 v-11}{4}} d s=\infty .
\end{gathered}
$$

Consequently, by Theorem 2.3, (3.6) is oscillatory.

Remark 3.2. The results obtained in this paper hold true if we replace condition $\left(H_{2}\right)$ by

$$
f \in C(\mathbb{R}, \mathbb{R}), \quad y f(y)>0 \quad \text { and } \quad \frac{f(y)}{y} \geq k>0 \quad \text { for } \quad y \neq 0,
$$

but the function $q(x)$ should be nonnegative in this case.

\section{ACKNOWLEDGEMENT}

The author would like to thank the referee for his valuable suggestions and useful comments. 


\section{REFERENCES}

1. D. Gilbarg and N. S. Trudinger, Elliptic partial differential equations of second order. Second edition. Grundlehren der Mathematischen Wissenschaften [Fundamental Principles of Mathematical Sciences], 224. Springer-Verlag, Berlin, 1983.

2. I. V. Kamenev, On the question of the oscillation of the solutions of a second order differential equation with an "integrally small" coefficient. (Russian) Differencial'nye Uravneniya 13(1977), No. 12, 2141-2148, 2300-2301.

3. E. S. Noussair and C. A. Swanson, Oscillation of semilinear elliptic inequalities by Riccati transformations. Canad. J. Math. 32(1980), No. 4, 908-923.

4. C. A. Swanson, Semilinear second-order elliptic oscillation. Canad. Math. Bull. 22(1979), No. 2, 139-157.

5. A. Wintner, A criterion of oscillatory stability. Quart. Appl. Math. 7(1949), 115-117.

6. Z. T. XU, Oscillation of solutions to second-order elliptic partial differential equations with a "weakly integrally small" coefficient. (Chinese) J. Systems Sci. Math. Sci. 18(1998), No. 4, 478-484.

7. Z. T. Xu, A Riccati technique and oscillation for semilinear elliptic equations. (Chinese) Chinese Ann. Math. Ser. A 24(2003), No. 5, 565-574; English transl.: Chinese J. Contemp. Math. 24(2003), No. 4, 329-340 (2004).

8. B. G. Zhang, T. ZhaO, and B. S. Lalli, Oscillation criteria for nonlinear second order elliptic differential equations. Chinese Ann. Math. Ser. B 17(1996), No. 1, 89-102.

(Received 28.12.2003)

Author's address:

Department of Mathematics

South China Normal University

Guangzhou 510631

P. R. China

E-mail: xztxhyyj@pub.guangzhou.gd.cn 